\title{
AN UNUSUALLY PROLONGED SURVIVAL IN METASTATIC PERIAMPULLARY PANCREATIC ADENOCARCINOMA
}

\author{
Shafaq Maqsood, Usman Ahmad \\ Department of Medical Oncology, Shaukat Khanum Memorial Cancer Hospital and Research Centre, Lahore, \\ Pakistan
}

Received: 30 September 2016 / Accepted: 1 December 2016

\begin{abstract}
Periampullary cancer is the term used for neoplasms arising from the head of pancreas, ampulla of Vater, distal bile duct and periampullary region of duodenum. Of these, the pancreatic adenocarcinoma carries the worst prognosis with majority of mortalities within the $1^{\text {st }}$ year. The overall 5 -year survival is $<3 \%$. Studies have shown that pancreatic carcinoma has the shortest median survival of 17.1 months of all periampullary carcinomas. With the development of metastasis, the median survival tends to reduce to 3-6 months. We are reporting a case of metastatic periampullary pancreatic adenocarcinoma that received minimal and interrupted chemotherapy due to poor tolerance and yet managed to survive far beyond the reported median survival.
\end{abstract}

Key words: Adenocarcinoma, pancreas, periampullary, prognosis

\section{Introduction}

Pancreatic adenocarcinoma is the most frequently occurring periampullary cancer, with an incidence of 9 in 100,000 cancers in the US annually. ${ }^{[1-3]}$

Obstructive jaundice is the form of presentation in $65-75 \%$ of patients while other presenting features may be pain, weight loss, anorexia, elevated liver enzymes and new-onset diabetes mellitus or pancreatitis. ${ }^{[2]}$ Tumour marker Ca 19-9 may be helpful in diagnosis which in $75 \%$ of cases is raised. Diagnostic imaging includes computed tomography (CT) scan, magnetic resonance imaging scan, endoscopic retrograde pancreatocholangiography (ERCP) and endoscopic ultrasound (EUS) which also aids in obtaining tissue for histological diagnosis. ${ }^{[2,4]}$

Treatment options include pancreatoduodenectomy with intent to cure, leading to increase in 5-year survival. Palliative options include palliative surgery, endoscopic stenting and/or chemotherapy. ${ }^{[4]}$ Locally advanced nonmetastatic periampullary pancreatic $\mathrm{Ca}$ has a median

Correspondence: Dr. Shafaq Maqsood, Department of Medical Oncology, Shaukat Khanum Memorial Cancer Hospital and Research Centre, Lahore, Pakistan.

Email: drshafaq@live.com survival of $6-10$ months. ${ }^{[5]}$ However, in metastatic settings, median survival is a mere $3-6$ months. ${ }^{[5,6]}$

Gemcitabine is the chemotherapeutic agent of choice in palliative settings which causes a modest but significant improvement in clinical condition and to some extent response and survival. ${ }^{[5,6]}$

In this paper, we present a case of an elderly lady diagnosed with metastatic periampullary pancreatic cancer who received suboptimal chemotherapy and managed to survive for 17 months after diagnosis. The case was chosen due to its unusual course and unexpectedly prolonged survival with minimal chemotherapy in metastatic settings.

\section{Case Report}

A 76-year-old female, known diabetic and hypertensive, presented to the gastroenterology clinic in September 2014, with a history of fever, weight loss and jaundice over the preceding 3 months. On examination, she was deeply jaundiced and emaciated.

Her initial workup done before presentation to the hospital included a CT scan of the abdomen obtained in August 2014, which showed biliary obstruction with suspicion of 
$4 \mathrm{~mm}$ non-calcified calculus at the level of ampulla. Her ERCP revealed periampullary pancreatic carcinoma and duodenal ulcer. Biopsy was taken which was positive for well-differentiated adenocarcinoma [Figure 1].

Her CT scan was repeated at our hospital which was suggestive of intra- and extra-hepatic biliary dilatation with dilated pancreatic duct traceable all the way up to the ampulla and presence of pulmonary metastasis

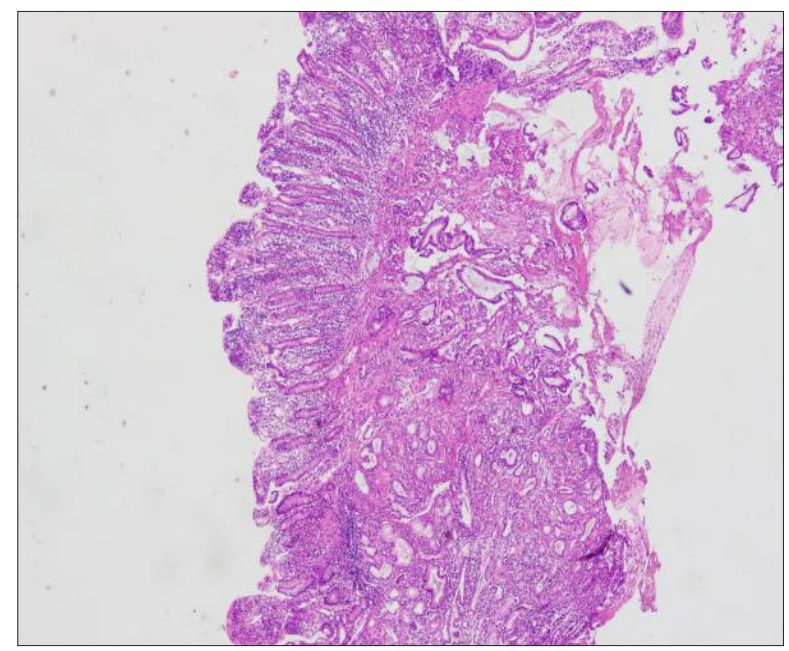

Figure 1: $(\times 4)$ A fragment of duodenal mucosa. The overlying epithelium shows ulceration and erosion with a dense inflammatory infiltrate. A tumour is seen infiltrating through the muscularis mucosae into the lamina propria

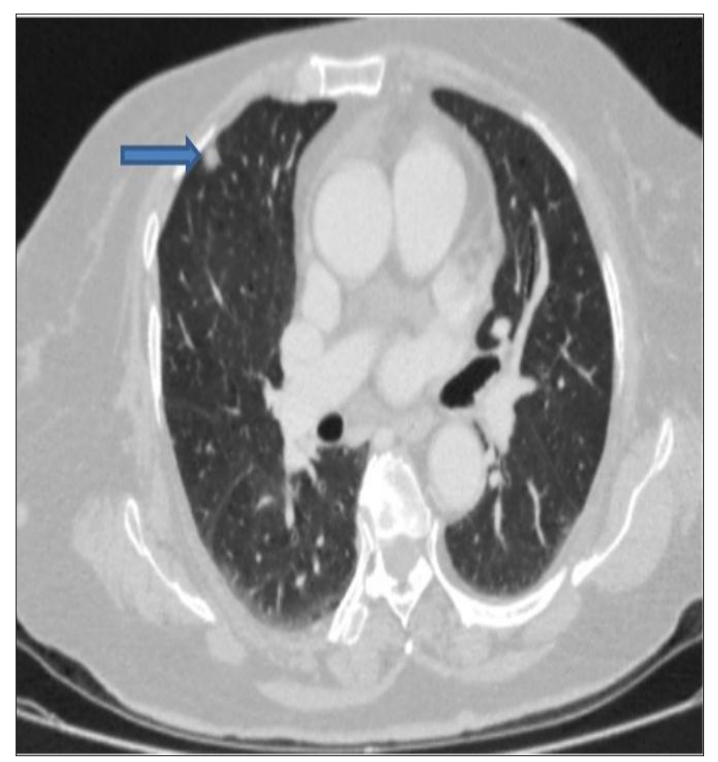

Figure 2: Baseline computed tomography scan showing lung metastasis (blue arrow) making it a Stage IV disease [Figure 2]. However, EUS was recommended for further assessment.

EUS was done which showed $25 \mathrm{~mm} \times 16 \mathrm{~mm}$ mass at the lower end of the common bile duct/in the periampullary region and a local node of $10 \mathrm{~mm}$. The patient was discussed in MDT and recommendations were ERCP with metallic stent insertion followed by chemotherapy gemcitabine with $25 \%$ dose reduction. The patient received two cycles of gemcitabine, but due to fatigue and anorexia, her regime was switched to capecitabine. After receiving one cycle of capecitabine, she was lost to followup. Her last visit to the hospital was in December 2014.

The patient resurfaced in October 2015 with fever, itching and vomiting, obstructive jaundice and raised lipase levels. She was treated on lines of cholangitis and pancreatitis with intravenous piptazobactam.

CT scan was repeated for restaging purposes and it showed progression in primary disease and appearance of hepatic metastasis [Figure 3].

Her blocked stent was replaced and she was discharged from the hospital in a stable clinical condition with improving liver function tests. She passed away in December 2015, 16 months after diagnosis and 12 months of no treatment for primary disease.

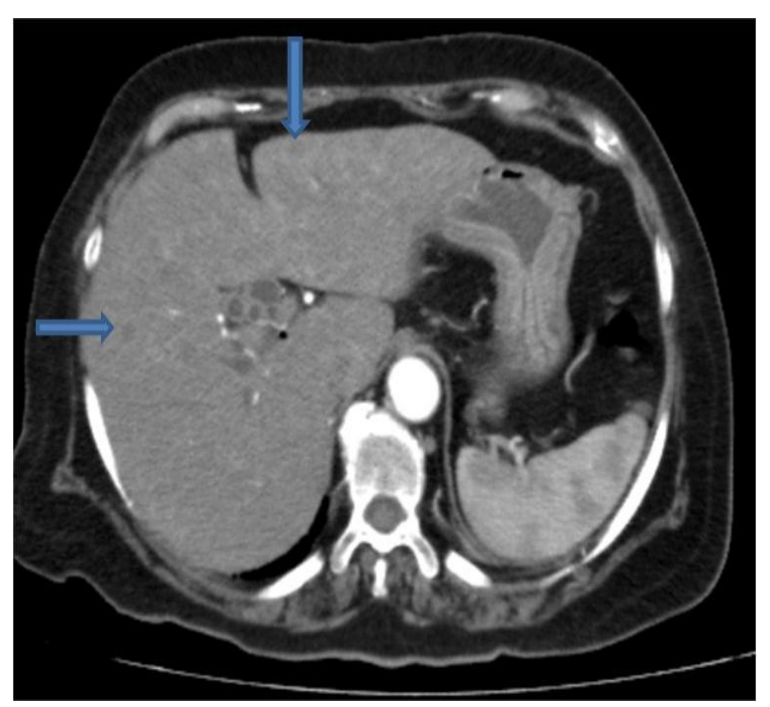

Figure 3: Computed tomography scan repeated after a year showed the presence of liver metastasis (blue arrows) consistent with disease progression 


\section{Discussion}

Periampullary tumours are defined as those tumours that arise within $2 \mathrm{~cm}$ of the major papilla in the duodenum and include pancreatic adenocarcinoma, distal bile duct and periampullary duodenal carcinomas..$^{[1,4,7]}$ Ampullary carcinomas lie in the region of ampulla of Vater and account for about $0.2 \%$ of tumours of the gastrointestinal tract and $6 \%$ of periampullary malignancies. ${ }^{[1,7]}$ Clinically, it is important to distinguish between ampullary and periampullary tumours due to the significance, it imparts to the prognosis and resectability. ${ }^{[1]}$ Ampullary carcinomas carry a better prognosis ${ }^{[1,7]}$ with 5 -year survival ranging from 34 to $45 \%{ }^{[1]}$

Most of the patients with pancreatic adenocarcinoma present at a late stage and carry a poor prognosis. ${ }^{[1]}$ Resectability is possible in only $15 \%$ of cases which increases the long-term survival in almost $25 \%$ of patients. ${ }^{[8]} 95 \%$ of patients usually die within a year and 5-year survival after a curative surgery is only 5-20\%, thus making pancreatic adenocarcinoma the worst of periampullary cancers. ${ }^{[1]}$ Metastatic periampullary pancreatic malignancy has a much lower median survival of 3-6 months. ${ }^{[6]}$

Commonly periampullary pancreatic adenocarcinoma presents with jaundice and abdominal pain. Other nonspecific symptoms are weight loss and anorexia. Newonset diabetes mellitus is also one form of presentation. ${ }^{[2,8]}$

Mostly periampullary tumours are adenocarcinomas. ${ }^{[4]}$ Radiological workup for pancreatic malignancy includes a CT scan which usually suggests the diagnosis in $95 \%$ of the cases. In periampullary tumours, endoscopic retrograde cholangiopancreatography is a part of diagnostic workup. It has a therapeutic role as well in case of obstructive symptoms with a stent placement. ${ }^{[8]}$ Endoscopic ultrasound has a role in staging workup by assessing the depth of invasion and involvement of peripancreatic lymph nodes along with obtaining fine-needle aspiration and brushings for cytology. ${ }^{[4]}$ Amongst the treatment modalities, surgical resection of periampullary malignancies is the only possible way of improving long-term survival; however, the resectability rate for pancreatic cancer is as low as $20 \%{ }^{[1]}$
Metastatic pancreatic cancer is associated with poor prognosis and low median survival of 3-6 months. Treatment of metastatic pancreatic cancer remains a challenge. The standard cytotoxic agent is gemcitabine. Although response rate is poor, it has shown clinical benefit in improving quality of life. ${ }^{[6,9]}$ Patients treated with gemcitabine have a median survival of 6.2 months and a 1-year survival of $20 \%{ }^{[10]}$ Combinations of gemcitabine with several other chemotherapeutic agents such as capecitabine, irinotecan, cisplatin or oxaliplatin have been studied in trials and have not shown any major advantage in survival. ${ }^{[9,10]}$

A combination of gemcitabine and epidermal growth factor tyrosine kinase inhibitor erlotinib has been approved by the Food and Drug Authority due to a significant longterm survival advantage. ${ }^{[10]}$

Review of literature clearly indicates a very low median survival associated with metastatic pancreatic periampullary malignancy even with chemotherapy. In case of our patient, she received interrupted chemotherapy and yet survived for 16 months after diagnosis and 12 months with no treatment whatsoever which was quite extraordinary. The prognosis for periampullary carcinoma in the resected patient is better than pancreatic adenocarcinoma, but in case of metastatic disease, the outcome is dismal. This particular case highlights the fact that there is indeed number of patients that might abate the need for cytotoxic chemotherapy and the related side effects. So far, there is/are no prognostic factor/s discovered which can be utilised to tailor treatment to each patient individually.

\section{Conflict of Interest}

The authors declare that they have no conflict of interest.

\section{References}

1. Fernandez-Cruz L. Peri ampullary carcinoma. In: Holzheimer RG, Mannick JA, editors. Surgical Treatment: Evidence-Based and Problem Oriented. Munich: Zuckschwerdt; 2001.

2. Sohn TA, Yeo CJ. Pancreatic and periampullary Carcinoma (non endocrine). In: Pancreas. Ch. 5. Vol. 3. San Diego, CA: Elsevier Health; 2004.

3. Hatzarus I, George N, Muscarella P, et al. Predictors of survival in periampullary cancers following 
pancreaticoduodenectomy. Ann Surg Oncol 2010;17:991-7.

4. Jagannath P, Shrikhande S. Current options in the diagnosis and management of periampullary carcinoma. Indian J Surg 2003;65:347-53.

5. Pancreatric Section, British Society of Gastroenterology, Pancreatic Society of Great Britain and Ireland, Association of Upper Gastrointestinal Surgeons of Great Britain and Ireland, Royal College of Pathologists, Special Interest Group for Gastro-Intestinal Radiology. Guidelines for the management of patients with pancreatic cancer periampullary and ampullary carcinomas. Gut 2005;54 Suppl 5:51-16.

6. Soldik Z, Zitnjak D, Bolanca A, et al. Long-time survival of a patient with metastatic pancreatic cancer: A case report. Case Rep Oncol 2011;4:367-70.
7. Klein F, Jacob D, Bahra M, et al. Prognostic factors for long-term survival in patients with ampullary carcinoma: The results of a 15-year observation period after pancreaticoduodenectomy. HPB Surg 2014;2014:970234.

8. Kuvshinoff BW, Bryer MP. Treatment of resectable and locally advanced pancreatic cancer. Cancer Control 2000;7:428-36.

9. El Kamar FG, Grossbard ML, Kozuch PS. Metastatic pancreatic cancer: Emerging strategies in chemotherapy and palliative care. Oncologist 2003;8:18-34.

10. Seufferlein T, Bachet JB, Cutsem EV, et al. Pancreatic adenocarcinoma: ESMO-ESDO clinical practice guidelines for diagnosis, treatment and follow up. Ann Oncol 2012;23:733-40. 